\title{
Translation and validation of two International Spinal Cord Injury (SCl) Data Sets-a modified process
}

\author{
Kerstin Wahman ${ }^{1,2} \cdot$ Erika Nilsson $^{3} \cdot$ Wolfram Antepohl $^{4} \cdot$ Kersti Samuelsson $^{4} \cdot$ Elisabet Åkesson $^{1,5}$. \\ André Kuhlefelt Sandberg ${ }^{6} \cdot{\text { Marika Augutis } \mathbb{D}^{1}}^{1}$
}

Received: 18 October 2019 / Revised: 4 December 2019 / Accepted: 4 December 2019

(c) The Author(s), under exclusive licence to International Spinal Cord Society 2019

\begin{abstract}
Study design A descriptive design was used of a reflective process of problem solving among individuals working together to improve the process of translating.

Setting Sweden.

Objectives The aim of this study was to describe a modified process for translation and validation of the International Spinal Cord Injury (SCI) Quality of Life (QoL) and Activity and Participation (A\&P) Basic Data Sets from English into Swedish. Methods The process of translation followed the Executive Committee for the International SCI Standards and Data Sets (ECSCI) recommendations. The initial translation was performed by translators. Experts in SCI were then assembled to scrutinize the translations and to reach a consensus for defining a final version.

Results The whole process was time consuming. To save time in future translations, the start-up planning is of great importance. To identify appropriate participants with knowledge and interest to be part of the project is crucial. In addition, the consensus meetings, when scrutinizing the translated International SCI Data Sets, should be well prepared and structured. We identified a few steps that could make the process more efficient.

Conclusions By adding a few steps as well as defining the role of a project coordinator, we believe that future translations of the International SCI Data Sets for non-English-speaking countries could be facilitated.
\end{abstract}

\section{Introduction}

Self-management, health care, and prevention of complications in the field of spinal cord injury (SCI) require structured and standardized measurments to describe

Marika Augutis

Marika.augutis@rvn.se

1 Department of Neurobiology, Care Sciences and Society, Division of Neurogeriatrics, Karolinska Institutet, Stockholm, Sweden

2 Rehab Station Stockholm/Spinalis R\&D Unit, Frösundaviks allé 4, SE-169 89 Solna, Sweden

3 Spinalis Foundation, Stockholm, Sweden

4 Department of Rehabilitation Medicine and Department of Medical and Health Sciences, Linköping University, Linköping, Sweden

5 Stockholms Sjukhem, R\&D Unit, Stockholm, Sweden

6 Department of Community Medicine and Rehabilitation, Umeå University, Umeå, Sweden changes in the patients' performance, function and/or participation over time. Systematic data collection may support self-management and standardized care/rehabiliation and facilitate international data exchange. Pooling of data is central for valid comparisons across sites and countries but also a way to increase the sample sizes in different studies, as the incidence of individuals with SCI is relatively low. To systematically collect reliable data and evidence is essential for knowing how to best prevent and treat SCI [1-3].

The International Society of Spinal Cord Injury (ISCoS) has since 2002 initiated standardization of the collection and reporting of a minimal amount of information about areas affecting an individual with SCI through the International SCI Data Sets [4]. Members of the Executive Committee of the International SCI Standards and Data Sets committees (ECSCI) have also collaborated with the National Institute of Neurological Disorders and Stroke (NINDS), National Institutes of Health, and common data element (CDE) to integrate the International SCI Data Sets with the other NINDS CDE resources [5]. 
Multinational research requires translations and cultural adaptation of included data sets when used in other than the source language (mostly English). There is also an increasing need for translations within each country, depending on languages spoken regionally and nationally and if there are immigrant populations, especially for selfreporting instruments.

Translation from the original document with the source language into the target language and adaptation into a new setting is time consuming and costly, but crucial for the development of quality and equality for persons with SCI in a global perspective.

Back-translation (i.e., when the original language is generated from the target language) is often recommended to highlight and identify unwanted discrepancies between the source document and the translated version. In recent years there has been a discussion if this method with back-translation is necessary, especially for questionnaires that are aimed for professional use in a specialized field [6].

ECSCI has recommended two alternative methods for translating data sets [7] such as (1) translation from the English version into the target language, followed by a back-translation into English, to confirm that the original meaning is preserved or (2) an initial translation performed by translators with knowledge about SCI, followed by a controlling process performed by "checkers", i.e. professionals with knowledge in SCI and the health concepts used.

In our work to translate the International SCI Quality of Life (QoL) [8] and Activity \& Participation (A\&P) [9] Basic Data Sets into Swedish, we used the latter method.

The aim of this report is to describe the further developed and modified process based on the experiences from translating and validating the International SCI QoL and A\&P Basic Data Sets from English into Swedish using one of the recommended translation methods described by ECSCI [7].

\section{Study design}

This study has a descriptive design. The reflective process of problem solving among individuals from varying professions and persons with their own experience of an SCI working together to improve the process of translation is described and discussed.

\section{Method}

The translation of the International SCI QoL [8] and A\&P [9] Basic Data Sets was performed as a Master of Science project at Umeå University together with Rehab Station Stockholm,
Spinalis Foundation, Karolinska Institutet, Stockholms Sjukhem, and Linköping University Hospital in Sweden.

\section{Description of the English version of the translated data sets}

To be useful in clinical settings, data sets must be brief and contain a minimum number of items that persons with an SCI as well as clinicians find necessary and valuable to record. Thus, the data sets are not comprehensive but consist of items defined by the working groups as the most important. Both data sets in the present study are intended to be used by clinicians; moreover, the International SCI A\&P Basic Data Set is not for self-reporting.

ECSCI recommends translations to emphasize and ensure conceptual equivalence instead of being a word-toword translation.

\section{The International SCI QoL Basic Data Set [8]}

The International SCI QoL Basic Data Set reflects subjective QoL. Each person assesses his or her own QoL from a personal perspective and utilizes his or her own internal standards and assessment to do so. In this assessment, people may consider all factors they feel contribute to their QoL, whether these are related to health, finances, family, or other factors.

The International SCI QoL Basic Data Set consists of three questions about satisfaction (during the last 4 weeks): general QoL (overall well-being), physical health, and psychological health. The scale ranges from 0 (completely dissatisfied) to 10 (completely satisfied) [8].

\section{The International SCI Activity and Participation Basic Data Set [9]}

The International SCI A\&P Basic Data Set consists of 8 items relating to mobility and self-care of the International Classification of Functioning Disability and Health (ICF) along with 16 items relating to domestic life, social interactions and relationships, major life areas and community, and social and civic life of the ICF. The International SCI A\&P Basic Data Set is composed of and adopted from the two already existing and validated (for adults with SCI) measures: Spinal Cord Independence Measure [10] for activity and Craig Handicap Assessment and Reporting Technique for participation [11].

\section{Procedure}

The translations of the International SCI QoL and A\&P Basic Data Sets were performed according to recommendations from the ECSCI [7]. 
Based on the ECSCI recommendations, we further defined steps to clarify the process for all involved, as described in Fig. 1.

The following steps were included:

(1) Permission to translate was requested and received by ECSCI.

(2) The initial translation from English into Swedish (version 2a) was performed by a master student (AKS) and physical therapist (RPT) and was supervised by one of the authors (MA), who is a research advisor, PhD, RPT. MA has experiences in SCI rehabilitation and research. Both AKS and MA have good knowledge in the English and Swedish language. A Communications Officer (EN) at the Spinalis Foundation who is not directly involved in the professional care did a parallel translation of the

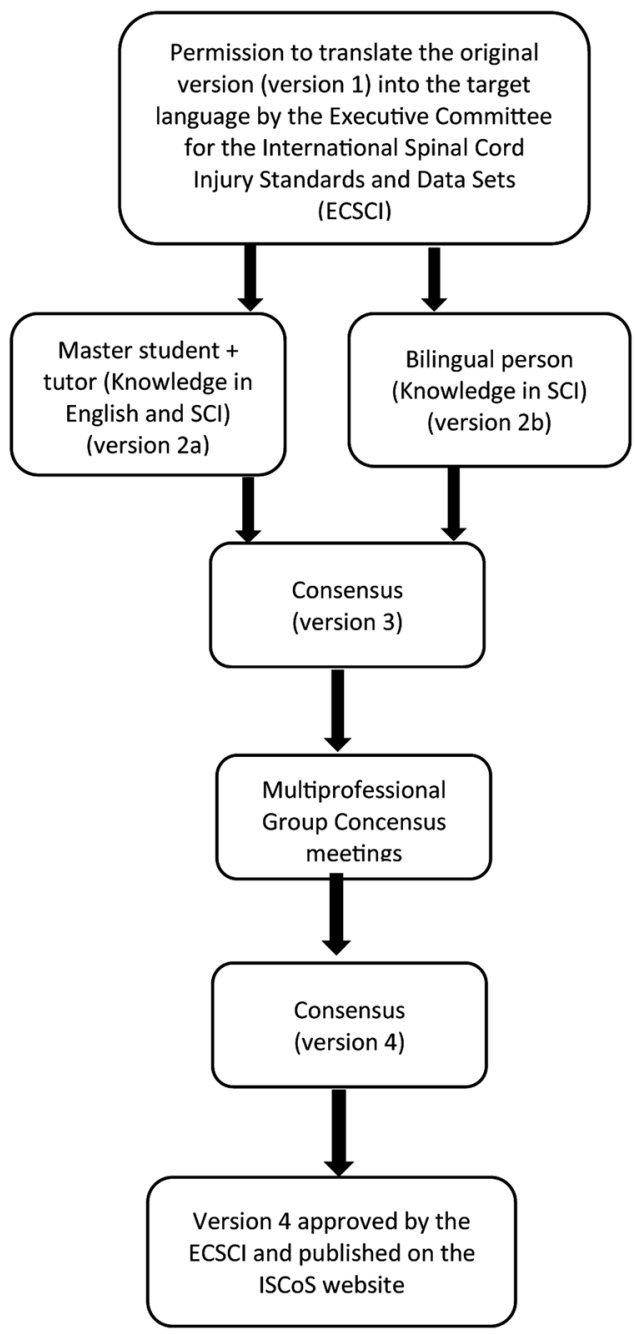

Fig. 1 The recommendation for translation according to the Executive Committee for the International SCI Standards and Data Sets (ECSCI) [7] interpreted by the consensus group. original version into Swedish (version $2 b$ ). EN has her own experience of living with an SCI and is bilingual.

(3) Versions $(2 a+2 b)$ were compared and discussed by AKS and MA, resulting in version 3 .

(4) Version 3 was mailed out to a group of SCI experts with a variety of clinical professions and SCI researchers, from Linköping and Stockholm.

(5) A first meeting was then scheduled with the expert group that had the following participants: one OT, one RPT, two MDs, and one social worker (also with own experience of living with an SCI), from the two major rehabilitation centers in Sweden; AKS, MA, and EN participated in the meeting. The meeting was face to face except for two professionals who attended through a video link. The translations were scrutinized and discussed several times until the group reached a consensus (an extra face-to-face meeting had to be scheduled to allow enough time for discussion), and the final Swedish version was set (version 4).

(6) The Swedish version of the International SCI QoL and A\&P Basic Data Sets was then sent to the ECSCI for approval and subsequently published on the ISCoS website: https://www.iscos.org.uk/international-sci-da ta-sets.

\section{Results}

\section{Experiences of the translation process}

Due to a lack of detailed information concerning the recommended translation process [7], the start-up planning took unnecessarily long time. It was also time consuming to find the appropriate participants who had both time and knowledge (both language and experience of SCI), and interest to participate in the project. Eight persons had to be engaged in the project. In addition, the consensus meetings, when scrutinizing the International SCI QoL and A\&P Basic Data Sets, were time consuming and an estimate was made that $\sim 5 \mathrm{~h}$ per participant were used for these meetings. A defined time plan for the project was a crucial omission in our process.

We have reflected on and discussed how to make the process more effective and suggest adding four new steps to the ECSCI recommended process (step numbers 5, 6, 7, and 9) (Fig. 2) in order to make the process clearer, be resource effective and time conscious regarding consensus meetings. We also believe that having a project coordinator $(\mathrm{PC})$ to keep the process clear and running is crucial.

Based on our experience, the following clarified $/ \mathrm{mod}$ ified process is suggested for future translations: 
Fig. 2 The suggested process of translation and validation of the International SCI Data Sets.

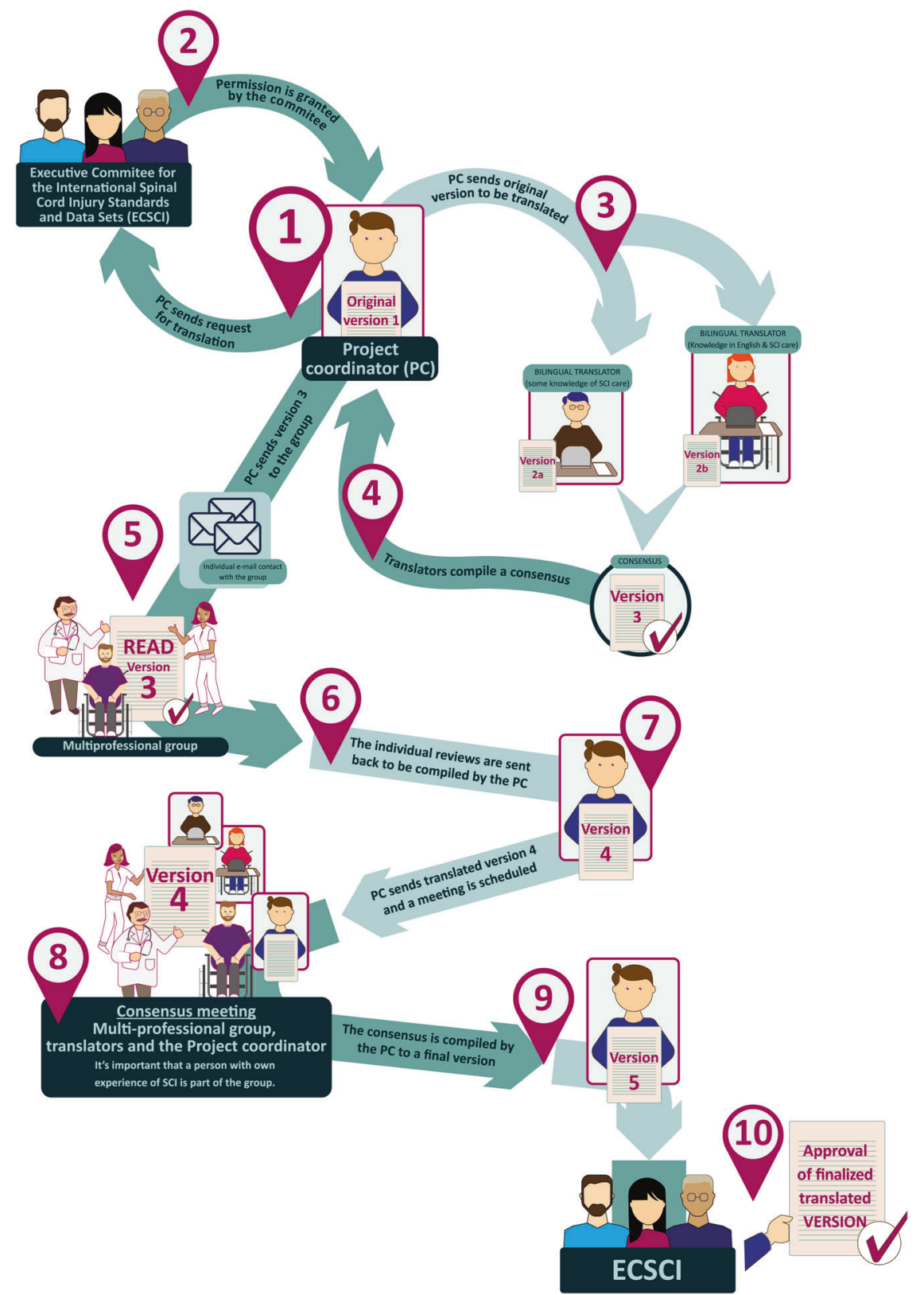

(1) A PC should be appointed who in turn contacts:

ECSCI and asks for permission to translate the data set.

(2) When permission is granted from the ECSCI, the PC contacts:

Two bilingual translators (knowing both the source language and the target language), where at least one of the translators also should have previous knowledge of SCI care or own experience of SCI. A group of professionals (experts in the field of the data set) and also at least one person with their own experience of SCI assembled, who all can set aside time needed to participate in the project according to the time schedule the PC has set for the whole process, with "dates to save" for all included participants.

(3) The PC sends the original English version (version 1) to the two translators and they make two independent versions $2 \mathrm{a}$ and $2 \mathrm{~b}$.

(4) Versions $2 \mathrm{a}$ and $2 \mathrm{~b}$ are then compared and compiled by the translators and the PC into version 3 .

(5) Version 3 is individually sent by the PC to the persons who are appointed to be members in the consensus group with a stated last date of return.

(6) The individuals' reviews of version 3 are then sent back to the PC who compiles the comments into version 4. If there are differences in the proposed translations, this is noticed and further discussed at a consensus meeting. 
(7) Version 4 is then sent back to the members of the consensus group and a meeting is scheduled. Version 4 then could include several versions of the proposed translation.

(8) The translation of the data set will then be scrutinized during a meeting until a consensus is reached. If disagreement(s) still exists that are not solved easily between version 3 (the initial translator's version) and the working group, ECSCI should be involved.

(9) The PC compiles the consensus into the final version (version 5) with approval by the members of the consensus group.

(10) Version 5 is then sent to the ECSCI for approval of a finalized translated version and subsequently published on the ISCoS website: https://www.iscos.org. uk/international-sci-data-sets.

\section{Discussion}

Common international data sets are considered essential to ensure quality in care, to improve care for the long-term perspective to develop life quality and participation for persons with SCI. Common data sets are a prerequisite for making data compiling and exchange possible.

One modifiable barrier for conducting international collaboration is the need for translated instrument. To date there are one International SCI Core Data Set, one Fracture Risk Data Set, and nineteen International SCI Basic Data Sets that need to be translated, several extended data sets, and then also additional questionnaires and surveys that are recommended by the ISCoS and the American Spinal Injury Association (ASIA) for describing the SCI population.

An effective translation process is of great importance for the instruments to be considered for use in self-reports, clinical care, and research. One of the key issues is to find individuals with relevant experience and competence, who can set aside time, and have good knowledge about English as well as about the situation, care, and rehabilitation of patients with SCI, to participate in this work.

Since English is the dominant language in the worldwide SCI communities (ISCoS and ASIA) who develop and provide SCI specific data sets, there is an extensive need for translation into other languages and cultural adaptations.

Experiences from the described translation process resulted in suggestions for an improved and more detailed stepwise process for the translation of the International SCI Data Sets. The upgraded process also includes a clear description of the role a PC should have in the process.

If the data sets are intended for self-reporting, they need to be further validated regarding usability and comprehendability both for the language and for the layout to make it easier to understand and to fill out.

We believe that a multiprofessional group with good knowledge of SCI care and rehabilitation, including a person with his or her own experience of SCI and also the source language (English), is the way to create the best conditions for the translation and cultural adaption and that using previous methods for translations (i.e., backtranslation into the source language) has been shown not to specifically improve translation quality [6].

\section{Limitations}

Our process could have been better planned at start, but we found it difficult to interpret the very brief recommendations that had been published [7]. The identified group was not well synchronized regarding requirements, expectations, time, and their own role, making the period before the actual translation could start time consuming and unnecessarily disorganized. When we started the translation process of the International SCI QoL and A\&P Basic Data Sets, we had no intention to document how much time the translators and each member of the consensus group used, which we later realized had been of interest to know. We have not yet had the opportunity to try or evaluate the suggested new process in clinical practice.

\section{Conclusions}

Resource effective translations of quantitative and qualitative instruments and data sets are crucial to follow and report on the status of persons with SCI over time and identify need for intervention. Furthermore, comparisons between SCI units in different countries with the goal to improve quality and equality as far as possible demand effective translation processes.

The process of translating data sets was here carefully described for the International SCI QoL and A\&P Basic Data Sets. By adding a few steps and defining a PC role, we believe that future translations and validation of the International SCI Data Sets into non-English languages will be facilitated. The translation of data sets to be used by persons with SCI, in clinical practice as well as research, is crucial to support individual self-management; local, national, and international comparison; research and development to reach quality; and equality goals.

Acknowledgements We gratefully thank Tamara Abarzúa Valencia who made the infographic. 


\section{Compliance with ethical standards}

Conflict of interest The authors declare that they have no conflict of interest.

Publisher's note Springer Nature remains neutral with regard to jurisdictional claims in published maps and institutional affiliations.

\section{References}

1. Biering-Sorensen F, Noonan V. Standardization of data for clinical use and research in spinal cord injury. Brain Sci. 2016;6:29. https://doi.org/10.3390/brainsci6030029.

2. Wyndaele M, Wyndaele JJ. Incidence, prevalence and epidemiology of spinal cord injury: what learns a worldwide literature survey? Spinal Cord. 2006;44:523-9.

3. Polinder S, Meerding WJ, Mulder S, Petridou E, van Beeck E, EUROCOST Reference Group. Assessing the burden of injury in six European countries. Bull World Health Organ. 2007;85:27-34.

4. Biering-Sørensen F, Charlifue S, DeVivo M, Noonan V, Post M, Stripling $\mathrm{T}$, et al. International Spinal Cord Injury Data Sets. Spinal Cord. 2006;44:530-4.

5. Biering-Sørensen F, Charlifue S, Devivo MJ, Grinnon ST, Kleitman N, Lu Y, et al. Incorporation of the International Spinal
Cord Injury Data Set elements into the National Institute of Neurological Disorders and Stroke Common Data Elements. Spinal Cord. 2011;49:60-4.

6. Epstein J, Osborne R, Elsworth G, Beaton D, Guillemin F. Crosscultural adaptation of the Health Education Impact Questionnaire: experimental study showed expert committee, not back-translation, added value. J Clin Epidemiol. 2015;68:360-9.

7. Biering-Sørensen F, Alexander MS, Burns S, Charlifue S, Devivo M, Dietz V, et al. Recommendations for translation and reliability testing of International Spinal Cord Injury Data Sets. Spinal Cord. 2011:49:357-60.

8. Charlifue S, Post MW, Biering-Sørensen F, Catz A, Dijkers M, Geyh S. et al. International Spinal Cord Injury Quality of Life Basic Data Set. Spinal Cord. 2012;50:672-5. https://doi.org/10. 1038/sc.2012.27.

9. Post MW, Charlifue S, Biering-Sørensen F, Catz A, Dijkers MP, Horsewell J, et al. Development of the International Spinal Cord Injury Activities and Participation Basic Data Set. Spinal Cord. 2016;54:530-4.

10. Catz A, Itzkovich M, Agranov E, Ring H, Tamir A. SCIMspinal cord independence measure: a new disability scale for patients with spinal cord lesions. Spinal Cord. 1997;35:850-6.

11. Whiteneck GG, Charlifue SW, Gerhart KA, Overhosler JD, Richardson GN. Quantifying handicap: a new measure of longterm rehabilitation outcomes. Arch Phys Med Rehabil. 1992;73:519-26. 